\title{
Trabeculotomy in a Behçet's Disease Patient One Week after Infliximab Administration
}

\author{
Aya Koike Tsuneaki Handa Masahiro Zako \\ Department of Ophthalmology, Aichi Medical University, Nagakute, Japan
}

Key Words

Infliximab · Behçet's disease · Trabeculotomy · Infection · Tumor necrosis factor-alpha

\begin{abstract}
Purpose: To describe a patient with Behçet's disease and anterior uveitis, which was not cured by local and systemic corticosteroid treatments, who underwent trabeculotomy one week after infliximab administration.
\end{abstract}

Methods: The patient received preoperative antibiotic therapy followed by trabeculotomy one week after infliximab administration. We observed ocular findings before and after surgery.

Results: Anterior uveitis improved after infliximab administration. The elevated intraocular pressure improved after surgery and there were no intraoperative complications. Neither ocular inflammatory attacks nor infectious complications were found in the operated eye of the patient during follow-up.

Conclusion: Trabeculotomy one week after administration of infliximab appears to be safe and effective in treating secondary glaucoma associated with Behçet's disease.

\section{Introduction}

Infliximab, a humanized antibody against tumor necrosis factor-alpha (TNF- $\alpha$ ), reduces uveitis attacks in patients with Behçet's disease (BD) [1,2], although anti-TNF$\alpha$ therapy increases the risk of infections due to the systemic blockade of TNF- $\alpha$ [3]. Cataract, glaucoma, or other vitreoretinal problems often accompany BD and patients may require intraocular surgery for treatment. However, it is not well understood whether infliximab increases the risk of infections associated with surgery or when the best time for intraocular surgery is after infliximab administration.

We recently examined the safety and effectiveness of infliximab administration one week before cataract surgery, and neither ocular inflammatory attacks nor infectious 
complications were found [4]. Here, we report on a male patient with BD and secondary glaucoma who successfully underwent trabeculotomy one week after his second preoperative infliximab administration without suffering any postoperative complications. Based on these small scale series, we suggest the appropriate timing for intraocular surgery in patients with BD may be one week after administration of infliximab.

\section{Case Report}

A 53-year-old Japanese man was referred to our department in July 2011 due to elevated intraocular pressure (IOP) with repeated anterior uveitis in his left eye since April 2011. He had lost sight in his right eye after recurrent uveitis and secondary glaucoma despite topical and systemic corticosteroid treatments and trabeculectomy performed three times in October 1998, December 2003, and November 2005. The ophthalmic examination disclosed that his left best-corrected visual acuity (BCVA) was decreased (20/100), while IOP was $36 \mathrm{~mm} \mathrm{Hg}$ in the right and $46 \mathrm{~mm} \mathrm{Hg}$ in the left eye. On slit-lamp examination, both corneas were edematous due to elevated IOPs, and inflammation was seen in the anterior chamber (cells $2+$, flare +, keratic precipitates (KP) +) and in the vitreous (cells $2+$ ) of the left eye. Fundus examination showed retinal vasculitis and increased cup-to-disc ratio in the left eye. The patient had a history of recurrent oral aphthae and erythema nodosa on his arms. The HLA-B51 antigen was present in the patient and he was diagnosed with BD.

The subject had already received topical treatment for uveitis and glaucoma of his left eye; therefore, we continued eye drops containing latanoprost $0.005 \%$, timolol maleate $0.5 \%$, dorzolamide hydrochloride $1 \%$, bunazosin hydrochloride $0.01 \%$, and betamethasone sodium phosphate $0.1 \%$. We started oral prednisolone (30 mg/day), which was gradually tapered as the anterior chamber inflammation disappeared after 3 weeks and IOP decreased to 10-15 mm Hg. Gonioscopy with a Goldman 3-mirror lens showed the angle of his left eye was open and there was slight peripheral anterior synechiae but no neovascularization. This patient was followed once at another clinic.

Four months later, this patient came to our department again because of elevated IOP (45-50 mm $\mathrm{Hg}$ ) with inflammation in the anterior chamber (cells 2+, flare 2+, KP 2+) and the vitreous (cells +) of his left eye (fig. 1). Oral prednisolone was increased (30 mg/day), but there was no improvement. We planned glaucoma surgery following infliximab administration. The computerized tomography to analyze his chest did not show an abnormal lesion, but his tuberculin reaction was positive; therefore, he started prophylactic isoniazid before infliximab administration.

Until the glaucoma surgery, this patient received intravenous D-mannitol $(500 \mathrm{ml})$ and acetazolamide $(500 \mathrm{mg})$ twice a day. Infliximab therapy $(5 \mathrm{mg} / \mathrm{kg})$ was intravenously administered at weeks 4 and 6 (fig. 1; week 0 was defined as the first day of the last uveitis attack). By the second infliximab administration, inflammation in the anterior chamber disappeared, although the systemic corticosteroid was gradually tapered. The patient underwent trabeculotomy one week after the second preoperative infliximab administration. Neither ocular inflammatory attacks nor infectious complications were found in the operated eye during postoperative follow-up. Third infliximab therapy was intravenously administered at week 10 (fig. 1) and every 8 weeks thereafter. The subject's left eye is currently in good condition without corneal edema and inflammation in the anterior chamber for the last four months. Typical glaucomatous visual field defects were detected by Goldman perimetry, but the patient's present left BCVA was 20/70 and the IOP was around $9 \mathrm{~mm} \mathrm{Hg.}$ A summary of changes in the left IOP and of treatments after the last uveitis attack are shown in figure 1. As in our previous reports, this patient received the following preoperative antibiotic therapy: $0.5 \%$ levofloxacin eye drops (Santen Pharmaceutical Company, Osaka, Japan) and $500 \mathrm{mg}$ levofloxacin oral tablets (Daiichi Pharmaceutical Company, Tokyo, Japan) [4, 5]. 


\section{Discussion}

The results from this case report demonstrate that trabeculotomy one week after administration of infliximab is safe and effective for a patient with BD. There were no adverse effects, such as infection. To our knowledge, there is only one published report describing glaucoma surgery in a patient with BD undergoing treatment with infliximab therapy [6]. In this previous report, three eyes underwent successful trabeculectomy, but the timing of the glaucoma surgery after the final preoperative infliximab administration was not described. In two reports describing cataract surgery in patients with BD receiving infliximab treatment $[7,8]$, patients underwent surgery about halfway through an eight-week interval between doses of infliximab. The serum levels of infliximab significantly correlate with its effectiveness in preventing recurrent episodes of uveitis [9], and this midpoint between infliximab doses may be appropriate for determining the timing of surgery, based on the risk of infection associated with drug concentration. Here, we performed trabeculotomy one week after the last preoperative infliximab administration without suffering any postoperative complications, including infection. It is expected that a high serum concentration of infliximab is maintained in this short period after administration; thus, we believe it to be a safer period for surgery than four weeks after infliximab administration in terms of preventing surgery-associated intraocular inflammation in BD patients.

After the last uveitis attack, the elevated IOP lasted more than 6 weeks in our patient and did not seem to be transient, which is associated with anterior uveitis. It is unclear whether this patient was a steroid responder. Although we performed trabeculotomy for this patient, we also planned trabeculectomy several weeks later if the initial trabeculotomy was not effective. Trabeculotomy was our first choice as there is a risk of postoperative infection in trabeculectomy, in spite of an excellent efficacy in reducing IOP. Trabeculectomy with mitomycin $\mathrm{C}$ has provided long-term safety and was effective in reducing IOP in cases with secondary glaucoma associated with BD [10, 11]. Trabeculectomy has also been successful in patients with BD treated with infliximab therapy [6]. However, as shown here, trabeculotomy is a good surgical option for secondary glaucoma associated with BD.

Fortunately, inflammation in the anterior chamber drastically improved after the initial infliximab administration without the use of other systemic drugs such as azathioprine, colchicine, and cyclosporin. This allowed us to promptly perform glaucoma surgery and to preserve stable IOP for several months after the surgery. This patient had already lost sight in his right eye the first time we met him; therefore, we considered the second uveitis attack as high risk for his left eye, and consequently, planned emergent treatment to protect it. Infliximab may be the first choice for treating urgent eye conditions in BD patients. However, if this patient still shows infliximabresistant uveitis, we may need to add other systemic drugs immediately.

\section{Disclosure Statement}

There is no proprietary interest, and no grants and funds were received in support of the study. 


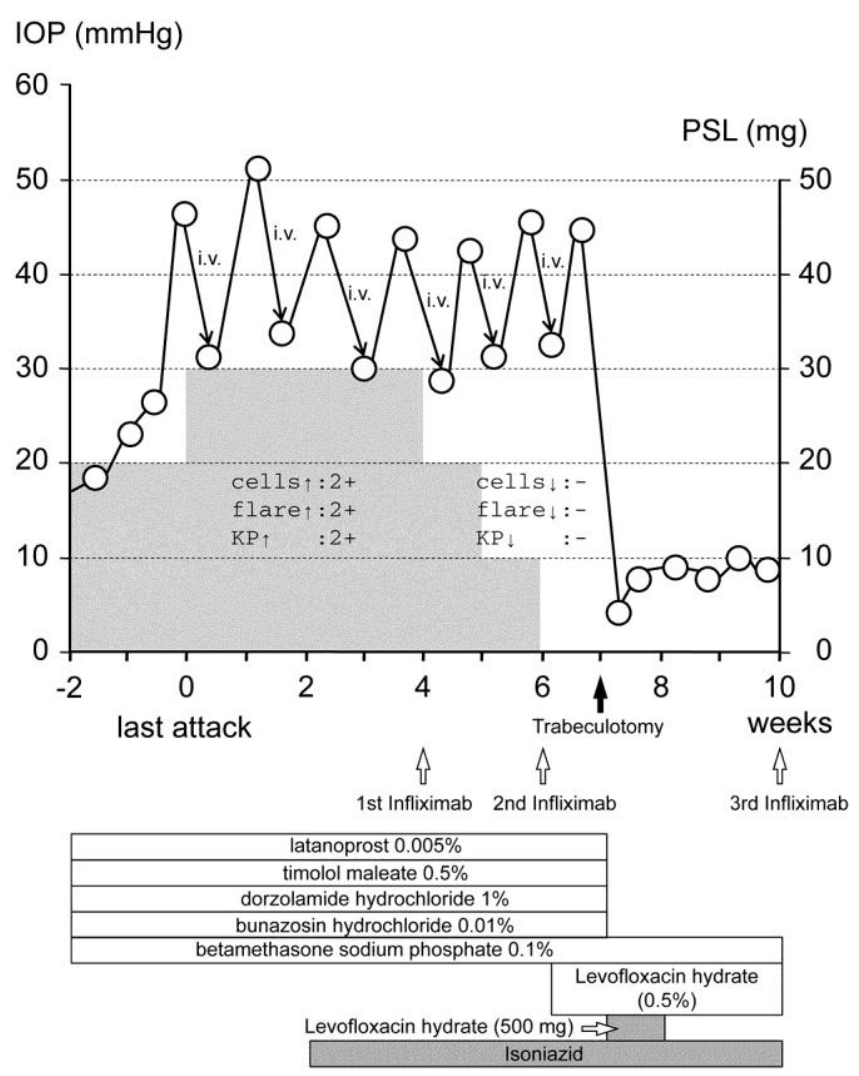

Fig. 1. Summary of the changes in the IOP and treatments for the last uveitis attack of the left eye. The first day of the last uveitis attack was defined as week 0 . A line graph with open circles demonstrates representative IOPs at each period. Anterior uveitis and elevated IOP were successfully treated after preoperative infliximab administration followed by trabeculotomy one week later. Inflammation in the anterior chamber is described. Oral administration of prednisolone, levofloxacin hydrate, and isoniazid are indicated by shading, and all other agents were topically administrated. Arrows indicate IOPs before and after intravenous administration of D-mannitol and acetazolamide. PSL = Oral prednisolone (mg/day); KP = keratic precipitates; i.v. = intravenous administration of Dmannitol (500 ml) and acetazolamide (500 mg) twice a day.

\section{References}

1 Sfikakis PP, Theodossiadis PG, Katsiari CG, Kaklamanis P, Markomichelakis NN: Effect of infliximab on sight-threatening panuveitis in Behçet's disease. Lancet 2001;358:295-296.

-2 Ohno S, Nakamura S, Hori S, Shimakawa M, Kawashima H, Mochizuki M, Sugita S, Ueno S, Yoshizaki K, Inaba G: Efficacy, safety, and pharmacokinetics of multiple administration of infliximab in Behçet's disease with refractory uveoretinitis. J Rheumatol 2004;31:1362-1368.

-3 Ellerin T, Rubin RH, Weinblatt ME: Infections and anti-tumor necrosis factor alpha therapy. Arthritis Rheum 2003;48:3013-3022.

4 Handa T, Tsunekawa H, Zako M: Cataract Surgery in Behçet's Disease Patients One Week after Infliximab Administration. Case Report Ophthalmol 2011;2:176-178.

-5 Ishida M, Kataoka T, Niwa K, Iwaki M, Zako M: Efficient penetration into aqueous humor by administration of oral and topical levofloxacin. J Ocul Pharmacol Ther 2011;27:247-250.

-6 Nishida T, Shibuya E, Asukata Y, Nakamura S, Ishihara M, Hayashi K, Takeno M, Ishigatsubo Y, Mizuki N: Clinical Course before and after Cataract and Glaucoma Surgery under Systemic Infliximab Therapy in Patients with Behçet's Disease. Case Report Ophthalmol 2011;2:189-192. 
7 Noda E, Yamanishi S, Shiraishi A, Ohashi Y: Cataract surgery under infliximab therapy in a patient with Behçet's disease. J Ocul Pharmacol Ther 2009;25:467-470.

8 Sakai T, Kanetaka A, Noro T, Tsuneoka H: Intraocular surgery in patients receiving infliximab therapy for Behçet disease. Jpn J Ophthalmol 2010;54:360-361.

-9 Sugita S, Yamada Y, Mochizuki M: Relationship between serum infliximab levels and acute uveitis attacks in patients with Behcet disease. Br J Ophthalmol 2011;95:549-552.

10 Yalvac IS, Sungur G, Turhan E, Eksioglu U, Duman S: Trabeculectomy with mitomycin-C in uveitic glaucoma associated with Behçet disease. J Glaucoma 2004;13:450-453.

11 Elgin U, Berker N, Batman A, Soykan E: Trabeculectomy with mitomycin C in secondary glaucoma associated with Behçet disease. J Glaucoma 2007;16:68-72. 